\title{
ON THE DERIVATIVE WITH RESPECT TO A POINT
}

\author{
A. W. GOODMAN
}

\begin{abstract}
The derivative of a polynomial $p(z)$ with respect to a point $\zeta$ is defined by the formula $A_{\varsigma} p(z)=(\varsigma-z) p^{\prime}(z)+n p(z)$, where $n$ is the degree of the polynomial. Let $p(z)$ have all its zeros in the unit disk and one zero at $z=1$. We determine a minimal region that must contain at least one zero of $A_{\varsigma} p(z)$.
\end{abstract}

1. Introduction. It is customary to define the derivative of a polynomial $p(z)$ with respect to a point $\zeta$ by the formula

$$
A_{\varsigma} \equiv A_{\varsigma}(p(z)) \equiv(\varsigma-z) p^{\prime}(z)+n p(z),
$$

where $n$ is the degree of the polynomial (see $[3$, vol. 1, p. 48; 4, vol. 2, p. 61]). Clearly $\lim A_{\varsigma}(p(z)) / \varsigma \equiv p^{\prime}(z)$ as $\varsigma \rightarrow \infty$. Thus $p^{\prime}(z)$ can be regarded as the derivative of $p(z)$ with respect to $\zeta=\infty$. Hence, theorems about $A_{\zeta}(p(z))$ can be regarded as generalizations for theorems about the derivative of $p(z)$.

An interesting conjecture due to Sendov runs as follows.

CONJECTURE. Let $p(z)$ be a polynomial with all of its zeros in $\bar{E}:|z| \leq 1$. If $a$ is any one of these zeros, then $p^{\prime}(z)$ has at least one zero in the disk $|z-a| \leq 1$.

This conjecture is still unsettled, although it is known to be true in many special cases. For details and further references see Schmeisser [6, 7]. One can always rotate the disk, and hence one can always assume that $0 \leq a \leq 1$ without loss of generality. Our interest centers on the special case that $a=1$, where we have

THEOREM A. Let $p(z)$ be a polynomial with all its zeros in $\bar{E}$ and assume that $p(1)=0$. Then $p^{\prime}(z)$ has at least one zero in $|z-1 / 2| \leq 1 / 2$ (see Goodman, Rahman, and Ratti [2] and Schmeisser [5]).

Here we consider the generalization of Theorem A to $A_{\varsigma}(p(z))$, the derivative of $p(z)$ with respect to the point $\zeta$. For each fixed $\zeta$ we obtain a minimal set in which $A_{\varsigma}$ must have at least one zero.

2. Statement of the result. If $\zeta=1$ and $p(1)=0$, then it is clear from (1.1) that $A_{\zeta}(p(z))=0$ when $z=1$. Thus the singleton set $\{1\}$ forms the minimal set when $\zeta=1$. Thus the case $\zeta=1$ is trivial. The case $\zeta=\infty$ can also be set aside because this is just the case settled by Theorem A. Henceforth we assume that $\varsigma \neq 1$ and $\varsigma \neq \infty$.

Received by the editors July 14, 1986.

1980 Mathematics Subject Classification (1985 Revision). Primary 30C10; Secondary 30C15.

Key words and phrases. Polynomials, zeros of the derivative, derivative with respect to a point, minimal set. 
THEOREM 1. Let $p(z)$ be a polynomial with all of its zeros in $\bar{E}$ and one zero at $z=1$. If $\varsigma \neq 1$ and $\varsigma \neq \infty$, then $A_{\varsigma}(p(z))$ has at least one zero in $\bar{K}$, where $\bar{K}$ is the image of $\bar{E}$ under the transformation

$$
w=L(z) \equiv \frac{(\varsigma-2) z+\varsigma}{-z+(2 \varsigma-1)} .
$$

This result is sharp. This means that $\bar{K}$ is a minimal set (if any point is deleted from $\bar{K}$, then the assertion is false).

The mapping (2.1) has several interesting properties. First, $L(\bar{E})=1$ iff $\zeta=1$; a trivial case that has already been discussed.

Second, $L(\bar{E})$ is the half-plane $\operatorname{Re} w \leq 1$ iff $\varsigma$ is on the circle $|\varsigma-1 / 2|=1 / 2$ and $\varsigma \neq 1$.

Third, $\bar{K}$ is always symmetric with respect to the real axis, and always contains the points $L(1)=1$ and $L(-1)=1 / \varsigma$.

Fourth, $\zeta$ is a fixed point of (2.1).

Finally, $L(\bar{E})=\bar{E}$ if and only if $|\varsigma|=1$ and $\varsigma \neq 1$.

3. Proof of Theorem 1. As in [2] we map $\bar{E}$ onto the half-plane $\operatorname{Re} w \geq 0$ by the Möbius transformation

$$
w=M(z)=\frac{1+z}{1-z}, \quad z=M^{-1}(w)=\frac{w-1}{w+1} .
$$

The equation $A_{\varsigma}(p(z))=0$ is satisfied whenever

$$
\frac{A_{\varsigma}(p(z))}{p(z)}=(\varsigma-z) \frac{p^{\prime}(z)}{p(z)}+n=0
$$

or whenever

$$
(\varsigma-z)\left(\frac{1}{z-1}+\sum_{k=1}^{n-1} \frac{1}{z-z_{k}}\right)+n=0 \text {. }
$$

Here we let $z_{1}, z_{2}, \ldots, z_{n-1}$ and $z_{n}=1$ be the zeros of $p(z)$. If $w_{k}=M\left(z_{k}\right)$ for $k=1,2, \ldots, n-1$ and $\varsigma^{*}=M(\varsigma)$, then the transformation (3.1) applied to (3.3) gives

$$
2 \frac{\varsigma^{*}-w}{\left(\varsigma^{*}+1\right)(w+1)}\left(\frac{w+1}{-2}+\sum_{k=1}^{n-1} \frac{(w+1)\left(w_{k}+1\right)}{2\left(w-w_{k}\right)}\right)+n=0 .
$$

After a few simple steps we arrive at

$$
-1+\sum_{k=1}^{n-1} \frac{w_{k}+1}{w-w_{k}}+n \frac{\varsigma^{*}+1}{\varsigma^{*}-w}=0 .
$$

We add 1 to each term under the sum sign, subtract $n$ from the last term, and divide by $w+1$. Here we observe that $w=-1=M(\infty)$. Then (3.5) gives

$$
\sum_{k=1}^{n-1} \frac{1}{w-w_{k}}-\frac{n}{w-\varsigma^{*}}=0
$$


Consequently if $\eta_{k}$ is any root of equation (3.6), it is also a root of (3.4), and $Z_{k}=M^{-1}\left(\eta_{k}\right)$ is a zero of $A_{\zeta}(p(z))$. We next put (3.6) in the form $N(w) / D(w)=0$, where $N$ and $D$ are polynomials, and find that

$$
N(w)=w^{n-1}+\left[(n-1) \varsigma^{*}-2\left(w_{1}+w_{2}+\cdots+w_{n-1}\right)\right] w^{n-2}+\cdots .
$$

Therefore if $\eta_{1}, \eta_{2}, \ldots, \eta_{n-1}$ are the roots of $N(w)=0$, then

$$
\eta_{1}+\eta_{2}+\cdots+\eta_{n-1}=2\left(w_{1}+w_{2}+\cdots+w_{n-1}\right)-(n-1) \varsigma^{*}
$$

Since $\operatorname{Re} w_{k} \geq 0$ for $k=1,2, \ldots, n-1$, equation (3.8) tells us that there exists at least one $\eta^{*}$ which is a root of $N(w)=0$, and for which

$$
\operatorname{Re} \eta^{*} \geq \operatorname{Re}\left(-\varsigma^{*}\right) \text {. }
$$

Then $Z_{c}=M^{-1}\left(\eta^{*}\right)$ is a root of $A_{\zeta}(p(z))=0$. Since (3.9) defines a half-plane $K^{*}: \operatorname{Re} w \geq \operatorname{Re}\left(-\varsigma^{*}\right)$, the image $\bar{K}$ of this half-plane under $z=M^{-1}(w)$ must contain at least one root or $A_{\zeta}(p(z))=0$.

Let $\eta^{*}$ be any fixed point in $K^{*}$, where $\varsigma$ and $\varsigma^{*}$ are fixed. We will prove that $\eta^{*}$ can occur as a zero of $N(w)$. To see this set $n=2$. Then equation (3.6) gives

$$
1 /\left(w-w_{1}\right)=2 /\left(w-\varsigma^{*}\right)
$$

and hence $w=\eta^{*}=2 w_{1}-\varsigma^{*}$. Thus by selecting $w_{1}$ suitably with $\operatorname{Re} w_{1} \geq 0$ we can force $\eta^{*}$ to be any preassigned point in $K^{*}$. Thus, the set $\bar{K}=M^{-1}\left(K^{*}\right)$ is a minimal set under the conditions of Theorem 1 . Unless $\zeta=1$, the set $\bar{K}$ is always a closed circular region, which may be a half-plane, or the closed exterior of a circle. Further, $\bar{K}$ is symmetric with respect to the real axis because it is the image of $\operatorname{Re} w \geq \operatorname{Re}\left(-\varsigma^{*}\right)$ under $M(w)=(w-1) /(w+1)$. Since the set $K^{*}$ is a minimal set for a root of (3.6), it follows that $\bar{K}$ is a minimal set for zeros of $A_{\zeta}(p(z))$. Finally, we observe that points of $\bar{K}$ occur for the quadratic $p(z)=(z-1)\left(z-z_{1}\right)$. For this polynomial we find that

$$
A_{\varsigma}(p(z))=\left(2 \varsigma-z_{1}-1\right) z-\left(\varsigma z_{1}-2 z_{1}+\varsigma\right) .
$$

Hence $A_{\varsigma}(p(z))=0$ for $z=z_{c}$, where

$$
z_{c}=\frac{(\varsigma-2) z_{1}+\varsigma}{-z_{1}+2 \varsigma-1}, \quad z_{1} \neq 2 \varsigma-1 .
$$

Thus as $z_{1}$ ranges over $\bar{E}$, equation (3.12) or $L(z)$ generates $\bar{K}$.

4. Some concluding remarks. Under the conditions of Theorem 1 , equation (2.1) provides the complete solution to the problem of finding a minimal set for some zero of $A_{\zeta}(p(z))$ when $p(z)$ is any polynomial with one zero at $z=1$ and all zeros in $\bar{E}$. Suppose, however, that we admit to competition only those polynomials of degree $n$ where $n$ is a fixed integer and $n>2$. Then a minimal set, which now depends on $n$, may be different from the one found in Theorem 1 . To illustrate this possibility, let us return to the original conjecture of Sendov, modified as follows.

PROBLEM. For fixed $n$, let $p(z)$ be a polynomial of degree $n$ with all of its zeros in $\bar{E}$ and one zero at $z=a$ where $a$ is fixed in $[0,1]$. Find a minimal set $S(n, a)$ that must always contain at least one zero of $p^{\prime}(z)$.

It is easy to prove that when $a=0$, one such minimal set is the disk

$$
|z| \leq(1 / n)^{1 /(n-1)} \text {. }
$$


Thus, in this case, $S(n, 0)$ is a strictly increasing sequence of regions as $n$ increases.

When $a=1$, a minimal set $S(n, 1)$ is known to be the disk $|z-1 / 2| \leq 1 / 2$ when $n=2$ or when $n=3$. The assertion is trivial when $n=2$ and the case $n=3$ was settled in [2]. As far as the author is aware the problem is still open for $n \geq 4$. Since Theorem A covers all polynomials, the set $S(n, 1)$ must be contained in the disk $|z-1 / 2| \leq 1 / 2$, but may in fact be smaller than this disk.

\section{REFERENCES}

1. A. W. Goodman, On the zeros of the derivative of a rational function, J. Math. Anal. Appl. (to appear).

2. A. W. Goodman, Q. I. Rahman, and J. S. Ratti, On the zeros of a polynomial and its derivative, Proc. Amer. Math. Soc. 21 (1969), 273-274.

3. E. Laguerre, Oeuvres, vol. 1, Gauthier-Villars, Paris, 1898.

4. G. Pblya and G. Szego, Aufgaben und Lehrsatze aus der Anlysis, Dover, New York, 1945.

5. G. Schmeisser, Bemerkungen zu einer Vermutung von Ilieff, Math. Z. 111 (1969), 121-125.

6. __ Zur Lage der Kritischen Punkte eines Polynoms, Rend. Sem. Mat. Univ. Padova 46 (1971), 405-415.

7. __ On Ilieff's conjecture, Math. Z. 156 (1977), 165-173.

Department of MAThematics, University of SOUTh Florida, TAMPA, Florida 33620 